\title{
VEGF-induced neuroprotection, neurogenesis, and angiogenesis after focal cerebral ischemia
}

\author{
Yunjuan Sun, Kunlin Jin, Lin Xie, Jocelyn Childs, Xiao Ou Mao, Anna Logvinova, \\ and David A. Greenberg
}

Buck Institute for Age Research, Novato, California, USA

\begin{abstract}
Vascular endothelial growth factor (VEGF) is an angiogenic protein with therapeutic potential in ischemic disorders, including stroke. VEGF confers neuroprotection and promotes neurogenesis and cerebral angiogenesis, but the manner in which these effects may interact in the ischemic brain is poorly understood. We produced focal cerebral ischemia by middle cerebral artery occlusion for 90 minutes in the adult rat brain and measured infarct size, neurological function, BrdU labeling of neuroproliferative zones, and vWF-immunoreactive vascular profiles, without and with intracerebroventricular administration of VEGF on days 1-3 of reperfusion. VEGF reduced infarct size, improved neurological performance, enhanced the delayed survival of newborn neurons in the dentate gyrus and subventricular zone, and stimulated angiogenesis in the striatal ischemic penumbra, but not the dentate gyrus. We conclude that in the ischemic brain VEGF exerts an acute neuroprotective effect, as well as longer latency effects on survival of new neurons and on angiogenesis, and that these effects appear to operate independently. VEGF may, therefore, improve histological and functional outcome from stroke through multiple mechanisms.
\end{abstract}

J. Clin. Invest. 111:1843-1851 (2003). doi:10.1172/JCI200317977.

\section{Introduction}

VEGF is an angiogenesis (1) and vascular permeability (2) factor that undergoes transcriptional and posttranscriptional induction by hypoxia; it couples hypoxia to angiogenesis in diverse tissues (3), including the brain (4). VEGF may have an important role in the vascular response to cerebral ischemia, because ischemia stimulates VEGF expression in the brain (5-9) and VEGF promotes the formation of new cerebral blood vessels (10, 11). Topical administration of VEGF to the surface of the brain reduces infarct size (12), intravenous VEGF improves neurological outcome (13), and intraventricular administration of an anti-VEGF Ab increases infarct size (14) after focal cerebral ischemia. These findings are consistent with a protective effect of VEGF, although it is unclear if angiogenesis is responsible. Moreover, cerebral vessels formed in response to VEGF are abnormally leaky $(9,13)$, which could exacerbate cerebral edema and worsen the outcome from ischemia. VEGF also exerts direct effects on neurons. For example, VEGF stimulates axonal outgrowth and improves the survival of cultured superior cervical

Received for publication January 28, 2003, and accepted in revised form April 9, 2003.

Address correspondence to: David A. Greenberg, Buck Institute for Age Research, 8001 Redwood Boulevard, Novato, California 94945, USA. Phone: (415) 209-2087; Fax:(415) 209-2230; E-mail: dgreenberg@buckinstitute.org.

Conflict of interest: The authors have declared that no conflict of interest exists.

Nonstandard abbreviations used: middle cerebral artery occlusion (MCAO); external carotid artery (ECA); middle cerebral artery (MCA); artificial cerebrospinal fluid (aCSF); doublecortin (Dcx); dentate gyrus (DG); subgranular zone (SGZ);

subventricular zone (SVZ). and dorsal-route ganglion neurons $(15,16)$ and enhances the survival of mesencephalic neurons in organotypic explant cultures (17). VEGF protects HN33 (mouse hippocampal neuron $\times$ neuroblastoma) cells from death induced by serum withdrawal (18) and reduces hypoxic death of both HN33 cells and cultured cerebral cortical neurons $(19,20)$. Additional evidence for direct neuronal protection by VEGF is that VEGF inhibits the death of cultured hippocampal neurons from glutamate (21) and $N$-methyl-D-aspartate (22) toxicity and that deletion of the hypoxia-response element from the VEGF promoter causes motor-neuron degeneration in mice (23). Because neuroprotective effects of other growth factors are associated with improved outcome from stroke, this could be true for VEGF as well.

Finally, VEGF has been implicated as a factor that promotes neurogenesis in the adult brain. One possibility is that this occurs through the establishment of a vascular niche that favors the proliferation and differentiation of neuronal precursors (24), perhaps by the release of brain-derived neurotrophic factor from endothelial cells (25). Alternatively, VEGF may exercise a direct mitogenic effect on neuronal precursors (26). Thus, VEGF expression in the ischemic brain could contribute to ischemia-induced neurogenesis (27) and modify outcome in that way as well.

We investigated the relationships among three effects of VEGF that might be important determinants of outcome from cerebral ischemia - neuroprotection, neurogenesis, and angiogenesis - in a rat model of transient focal ischemia induced by middle cerebral artery occlusion (MCAO). Because previous evidence indicated that VEGF improves neurological recovery 
when given 48 hours, but not 1 hour, after ischemia (13), we delayed VEGF administration (1-3 days after ischemia) in these studies as well. Our results suggest that VEGF exerts multiple, independent effects on the ischemic brain that may modify both short-term and long-term outcome after stroke.

\section{Methods}

Focal cerebral ischemia. Animal experiments were approved by local committee review and were conducted according to the NIH Guide for the Care and Use of Laboratory Animals. Every effort was made to minimize animal suffering and to reduce the number of animals used. Transient focal cerebral ischemia was induced using the suture occlusion technique (28). Male Sprague-Dawley rats weighing 280-310 g were anesthetized with $4 \%$ isoflurane in $70 \% \mathrm{~N}_{2} \mathrm{O}$ and $30 \%$ $\mathrm{O}_{2}$, using a mask. A midline incision was made in the neck, the right external carotid artery (ECA) was carefully exposed and dissected, and a 3-0 monofilament nylon suture was inserted from the ECA into the right internal carotid artery to occlude the origin of the right middle cerebral artery (MCA). After 90 minutes of occlusion, the suture was removed to allow reperfusion, the ECA was ligated, and the wound was closed. Sham-operated rats underwent identical surgery except that the suture was not inserted. Rectal temperature was maintained at $37.0 \pm 0.5^{\circ} \mathrm{C}$ using a heating pad and heating lamp. Blood pressure and blood glucose concentration were monitored. Rats were killed at various times after reperfusion: they were anesthetized and perfused through the heart with 4\% paraformaldehyde in PBS ( $\mathrm{pH} 7.4$ ), the brains were removed, and brain sections were stained for histological evaluation.

Neurological assessment. Neurological evaluations were carried out 30 minutes after the onset of reperfusion and at various times thereafter, immediately before rats were killed for histological studies, using a modified behavioral rating scale (neurological severity score). This score is derived by evaluating animals for hemiparesis (response to raising the rat by the tail or placing the rat on a flat surface), abnormal movements (immobility, tremor, seizures), sensory deficits (placing, proprioception), and absent reflexes (pinna, corneal, startle), as described in detail (see ref. 29). Severe injury is reflected by a score of $10-14$, moderate injury 5-9, and mild injury 1-4.

Quantification of infarct volume. After reperfusion for various periods, rats were anesthetized with isoflurane, decapitated, and transcardially perfused with normal saline and then a $4 \%$ phosphate-buffered formaldehyde solution. Brains were carefully removed, fixed in formaldehyde, and dehydrated in phosphate-buffered 25\% sucrose. Cryopreserved 25$\mu \mathrm{m}$ coronal sections were stained with hematoxylin. Infarct area, left hemisphere area, and total brain area were measured on each 20 th section by a blinded observer using the NIH Image program, and areas were multiplied by the distance between sections to obtain the respective volumes. Infarct volume was calculated as a percentage of the volume of the contralateral hemisphere, as described (30).

VEGF administration and BrdU labeling in vivo. One day after induction of ischemia, rats were anesthetized with $4 \%$ isoflurane in $70 \% \mathrm{~N}_{2} \mathrm{O} / 30 \% \mathrm{O}_{2}$ and implanted with an osmotic minipump (Alzet 1003D; Alza Scientific Products, Mountain View, California, USA). The cannula was placed in the left lateral ventricle $4.0 \mathrm{~mm}$ deep as measured from the pial surface, $0.8 \mathrm{~mm}$ anterior to the bregma, and $1.3 \mathrm{~mm}$ lateral to the midline. VEGF $(10 \mu \mathrm{g} / \mathrm{ml})$ was administered at 1 $\mu \mathrm{l} / \mathrm{h}$ by the intracerebroventricular route for 3 days, beginning 24 hours after ischemia, in artificial cerebrospinal fluid (aCSF) consisting of $128 \mathrm{mM} \mathrm{NaCl}$, $2.5 \mathrm{mM} \mathrm{KCl}, 0.95 \mathrm{mM} \mathrm{CaCl}_{2}$, and $1.99 \mathrm{mM} \mathrm{MgCl}_{2}$. Control rats received aCSF without VEGF for the same amount of time. BrdU (50 mg/ $/ \mathrm{kg}$ in saline) or saline was given twice daily by the intraperitoneal route for the same period. At various times thereafter, rats were perfused with saline, and brains (four per condition) were fixed in $4 \%$ paraformaldehyde in PBS. For immunocytochemistry, $50-\mu \mathrm{m}$ coronal sections were treated with $50 \%$ formamide $/ 280 \mathrm{mM}$ $\mathrm{NaCl} / 30 \mathrm{mM}$ sodium citrate at $65^{\circ} \mathrm{C}$ for 2 hours, incubated in $2 \mathrm{M} \mathrm{HCl}$ at $37^{\circ} \mathrm{C}$ for 30 minutes, and rinsed in $0.1 \mathrm{M}$ boric acid ( $\mathrm{pH} 8.5$ ) at room temperature for 10 minutes. Sections were then incubated in $1 \% \mathrm{H}_{2} \mathrm{O}_{2}$ in PBS for 15 minutes, in blocking solution ( $2 \%$ goat serum $/ 0.3 \%$ Triton $\mathrm{X}-100 / 0.1 \%$ BSA in PBS) for 2 hours at room temperature, and with $2 \mu \mathrm{g} / \mathrm{ml}$ mouse monoclonal anti-BrdU (Roche Applied Science, Indianapolis, Indiana, USA) at $4{ }^{\circ} \mathrm{C}$ overnight. Sections were washed with PBS, incubated with biotinylated goat-anti-mouse secondary Ab (1:200; Vector Laboratories, Burlingame, California, USA) for 2 hours at $25^{\circ} \mathrm{C}$, washed, and placed in avidinperoxidase conjugate solution (Vector Laboratories) for 1 hour. The horseradish-peroxidase reaction was detected with $0.05 \%$ diaminobenzidine and $0.03 \%$ $\mathrm{H}_{2} \mathrm{O}_{2}$. Processing was stopped with $\mathrm{H}_{2} \mathrm{O}$, and sections were dehydrated through graded alcohols and cleared in xylene, and coverslips were applied using permanent mounting medium (Vector Laboratories). Sections were examined with a Nikon E300 epifluorescence microscope.

Immunocytochemistry. Rat brain sections were processed for immunocytochemistry and doublelabel immunocytochemistry as described (26). For double-label immunocytochemistry, sections were fixed with 4\% paraformaldehyde in PBS for 1 hour at room temperature, washed twice with PBS, and incubated in $2 \mathrm{M} \mathrm{HCl}$ at $37^{\circ} \mathrm{C}$ for 1 hour. Sections were incubated in blocking solution with primary Ab's at $4^{\circ} \mathrm{C}$ overnight and with secondary Ab's at room temperature for 2 hours. Primary Ab's were as follows: mouse monoclonal anti-BrdU $(2 \mu \mathrm{g} / \mathrm{ml}$; Roche Applied Science); affinity-purified goat polyclonal 


\section{Table 1}

Physiological measurements before and 30 minutes after the onset of MCAO in rats pretreated with aCSF vehicle or VEGF

\begin{tabular}{lcc}
\hline & Control & VEGF \\
$\begin{array}{l}\text { MABP }(\mathrm{mmHg}) \\
\text { Baseline }\end{array}$ & \\
30 minutes & $111 \pm 11$ & $116 \pm 9$ \\
$\mathrm{PaO}_{2}(\mathrm{mmHg})$ & $116 \pm 9$ & \\
Baseline $_{30 \text { minutes }}$ & $86 \pm 6$ & $84 \pm 6$ \\
$\mathrm{PaCO}_{2}(\mathrm{mmHg})$ & $83 \pm 5$ & $85 \pm 5$ \\
Baseline & & \\
30 minutes & $59 \pm 4$ & $56 \pm 4$ \\
pH & $56 \pm 4$ & $58 \pm 5$ \\
Baseline & & \\
30 minutes & $7.41 \pm 0.94$ & $7.37 \pm 1.36 \pm 1.15$ \\
Glucose (mg/dl) & $7.39 \pm 1.22$ & $175 \pm 11$ \\
Baseline & & $172 \pm 12$ \\
30 minutes & $178 \pm 20$ & \\
\hline
\end{tabular}

Control (treated with aCSF vehicle) or VEGF, $n=3 . \mathrm{PaO}_{2}$, arterial pressure of oxygen; $\mathrm{PaCO}_{2}$, arterial pressure of carbon dioxide.

anti-NeuroD (1:100; Santa Cruz Biotechnology Inc., Santa Cruz, California, USA); affinity-purified goat polyclonal anti-doublecortin (Dcx; 1:100) (Santa Cruz Biotechnology Inc.); rabbit polyclonal anti-cleaved (17-20 kDa) caspase-3 (1:500; New England Biolabs Inc., Beverly, Massachusetts, USA); and rabbit polyclonal anti-vWF (1:500; Sigma-Aldrich, St. Louis, Missouri, USA). Biotinylated goat anti-mouse IgG and FITC-conjugated (Vector Laboratories) and rhodamine-conjugated (Jackson ImmunoResearch) secondary Ab's were used (all were 1:200). Fluorescence signals were detected with a Nikon E300 or E800 microscope at excitation/emission wavelengths of 535/565 nm (rhodamine: red) and 470/505 nm (FITC: green). Biotin signals were detected with diaminobenzidine. Results were recorded with a Magnifire digital camera (ChipCoolers Inc., Warwick, Rhode Island, USA). Controls included omitting or preabsorbing primary $\mathrm{Ab}$ and omitting secondary $\mathrm{Ab}$. For confocal microscopy, a Nikon PCM-2000 laser-scanning

\section{Figure 1}

Effects of intraventricular VEGF treatment on infarct size and cell damage after MCAO. Rats underwent MCAO and were treated with intraventricular aCSF (MCAO) or VEGF (MCAO + VEGF), and infarct size was measured on cresyl violet-stained brain sections (a). In the cerebral cortical region salvaged by VEGF (i.e., that region stained intensely with cresyl violet in VEGF- but not aCSF-treated rats; indicated by arrow in top right), higher magnification showed that VEGF increased the number of cells with normal neuronal morphology and decreased the number of shrunken and misshapen cells in cresyl violet-stained sections $(\mathrm{CV})(\mathbf{b})$, reduced DNA-strand breaks labeled by the Klenow fragment of DNA polymerase I (Klenow) (c), and decreased caspase- 3 cleavage detected by staining with an Ab against its 17 - to $20-\mathrm{kDa}$ cleavage product (Caspase-3) (d). Data shown are representative of findings from at least four animals per experiment. confocal microscope and Simple PCI imaging software (Compix Inc., Cranberry Township, Pennsylvania, USA) were used.

Image analysis. To quantify angiogenesis after MCAO with or without VEGF treatment, images ( $\times 100$ magnification) of the dentate gyrus (DG) or the striatal ischemic penumbra region were acquired using a Nikon Eclipse-800 microscope and Nikon digital camera DXM1200 and software. The vWFimmunoreactive area in each image was determined by image analysis using Simple PCI software (Compix Inc.). In each experiment, areas of immunoreactivity were identified in the control sample and used to define representative ranges of values for brown pixels. Using these defined parameters, fields from each experiment were collected, and the images were analyzed by the PCI image analysis software, which calculated the total area covered by pixels, matching the defined intensities for the brown color component. The values corresponding to total brown areas were averaged and expressed as the mean percentage of stained vessel area per $100 \mu \mathrm{m}^{2}$.

DNA-damage (Klenow) assay. DNA single- and doublestrand breaks were determined using Klenow assays (31). Sections were fixed with $4 \%$ paraformaldehyde in PBS and treated with $1 \%$ Triton X-100 for 30 minutes and with $2 \%$ hydrogen peroxide for 15 minutes.

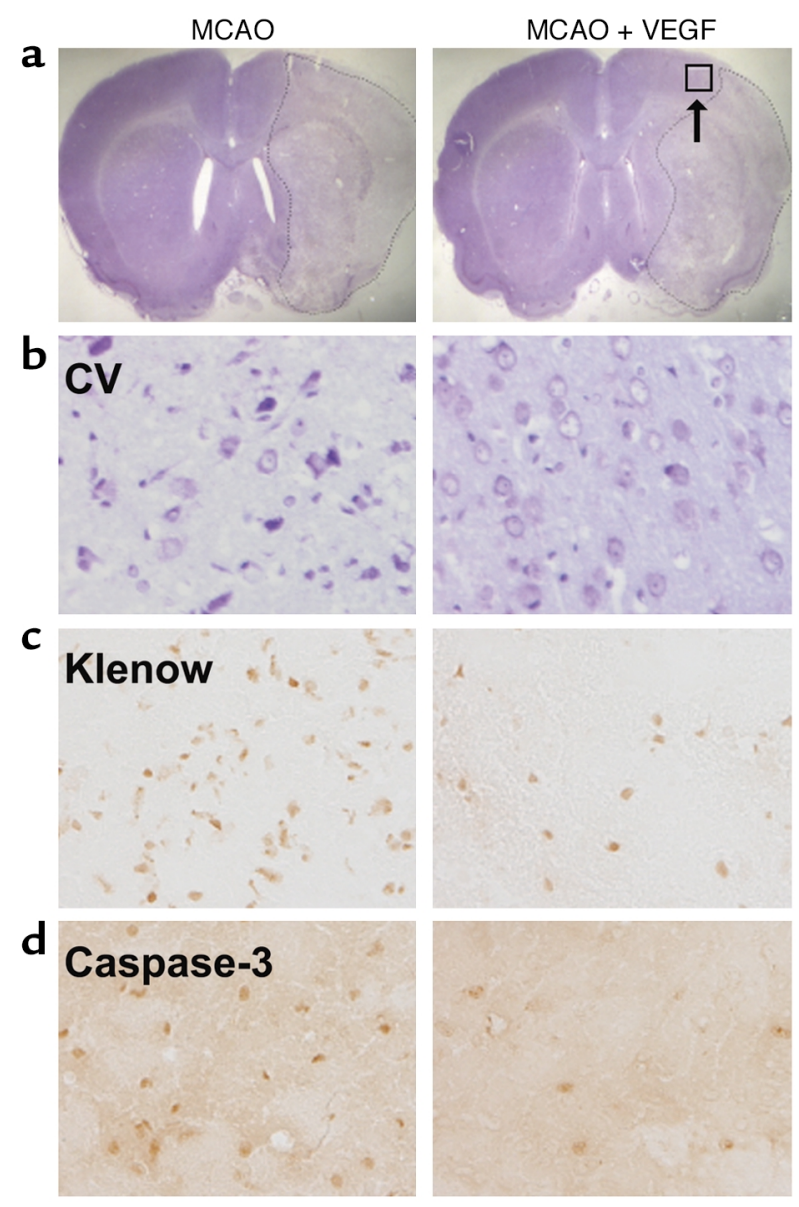



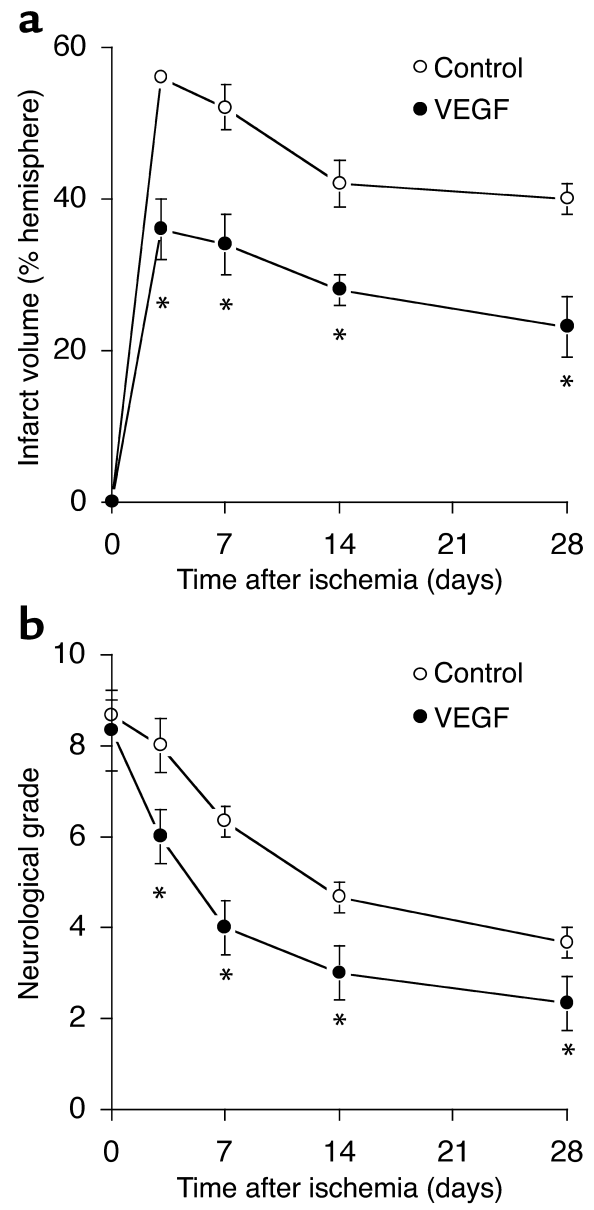

Sections were incubated in a moist-air chamber at $37^{\circ} \mathrm{C}$ for 1 hour in $60 \mu \mathrm{M}$ each of dGTP, dCTP, and dTTP; $59 \mu \mathrm{M}$ biotinylated dATP; $1 \mu \mathrm{M} \mathrm{dATP}$; and 40 $\mathrm{U} / \mathrm{ml}$ of Escherichia coli Klenow fragment in $1 \times$ reaction buffer (50 mM Tris- $\mathrm{HCl}, \mathrm{pH} 8.0,10 \mathrm{mM} \mathrm{MgCl}_{2}, 50$ $\mathrm{mM} \mathrm{NaCl}$ ). After washing, slides were incubated with streptavidin-horseradish peroxidase (VECTASTAIN Elite ABC; Vector Laboratories) for 1 hour at room temperature. The biotin-streptavidin-peroxidase complex was detected with $0.5 \mathrm{mg} / \mathrm{ml}$ diaminobenzidine and $0.05 \% \mathrm{H}_{2} \mathrm{O}_{2}$. To determine nonspecific labeling, selected sections were incubated in the reaction buffer without the Klenow fragment.

Statistics. Experiments were repeated at least three times. For quantitative data, the statistical significance of differences between means was evaluated by ANOVA or by two-way ANOVA with post hoc Tukey tests, as appropriate.

\section{Results}

VEGF and neuroprotection in ischemic brain. Rats that underwent MCAO followed by intraventricular infusion of VEGF were unaffected, compared with rats that underwent MCAO followed by aCSF, with respect to mean arterial blood pressure, arterial partial pressure of $\mathrm{O}_{2}$ or $\mathrm{CO}_{2}$, arterial blood $\mathrm{pH}$, and blood glucose concentration (Table 1). Cerebral infarcts in VEGF-treated

\section{Figure 2}

Quantitative effects of intraventricular VEGF on infarct volume and neurological function after MCAO. Rats were subjected to MCAO and treated with intraventricular aCSF (Control) or VEGF, and infarct volume (a) and neurological grade (b) were determined at the intervals shown. Note that a higher neurological grade in $\mathbf{b}$ denotes more severely impaired function. Two-way ANOVA showed significant effects of treatment (Fisher ratio $[\mathrm{F}]=68.25, P<0.0001$ ) and time $(\mathrm{F}=9.33, P=0.001)$ on infarct volume and of treatment $(\mathrm{F}=30.25$, $P<0.0001)$ and time $(\mathrm{F}=27.41, P<0.0001)$ on neurological grade, but no interaction between treatment and time $(F=0.42, P=0.741)$. ${ }^{*} P<0.01$ compared with control on same day (Tukey test; $n=3$ ).

rats were reduced in size, however, especially regarding the extent to which they involved the dorsomedial cortex in the region of the anterior cerebral artery/MCA border zone, or penumbra (Figure 1). Within this region, VEGF treatment was associated with a reduction in cytopathological features of cell injury, including cell shrinkage, DNA-strand breaks, and caspase-3 cleavage. Infarct volume at 3 days was reduced approximately $35 \%$ in VEGF-treated rats, and this difference persisted for at least 4 weeks (Figure 2a), indicating that VEGF decreased, and did not simply delay, tissue injury. Over the same 4-week interval, rats that received VEGF displayed consistently better neurological function, and the magnitude of this difference also remained constant over time (Figure $2 \mathrm{~b}$ ). Thus, the effect of VEGF on neurological grade scoring was manifested within the first 3 days after ischemia and persisted thereafter. Neurological improvement in VEGFtreated rats was attributable primarily (64\% of the total decrease in neurological severity score) to improvement in two motor tests (head movement upon raising the rat by the tail and circling behavior toward the paretic side upon placing the rat on the floor) and one sensory test (proprioception). In contrast, there was little difference between treated and untreated rats with respect to abnormal movements or reflex function.
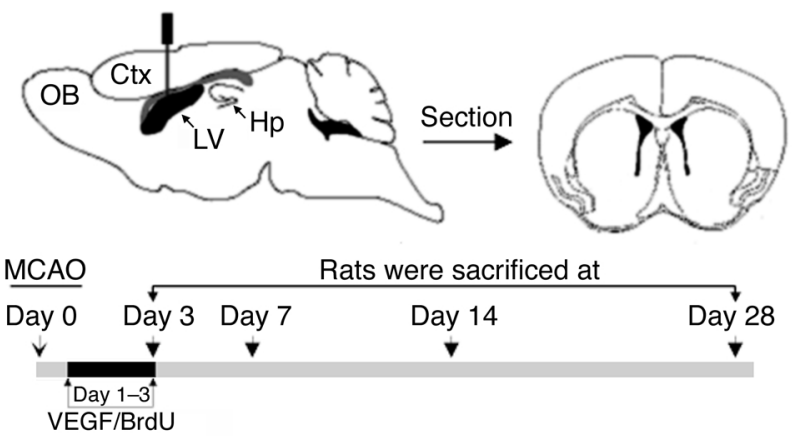

Figure 3

Scheme for quantifying postischemic neurogenesis in the rat brain. MCAO was induced on day 0 , VEGF (given by the intracerebroventricular route as shown in upper left) and $\mathrm{BrdU}$ (given by the intraperitoneal route) were administered on days $1-3$, and rats were killed and brains sectioned for BrdU immunohistochemistry and cell counting on days $3,7,14$, and 28 . OB, olfactory bulb; Ctx, cerebral cortex; LV, lateral ventricle; $\mathrm{Hp}$, hippocampus. 
a

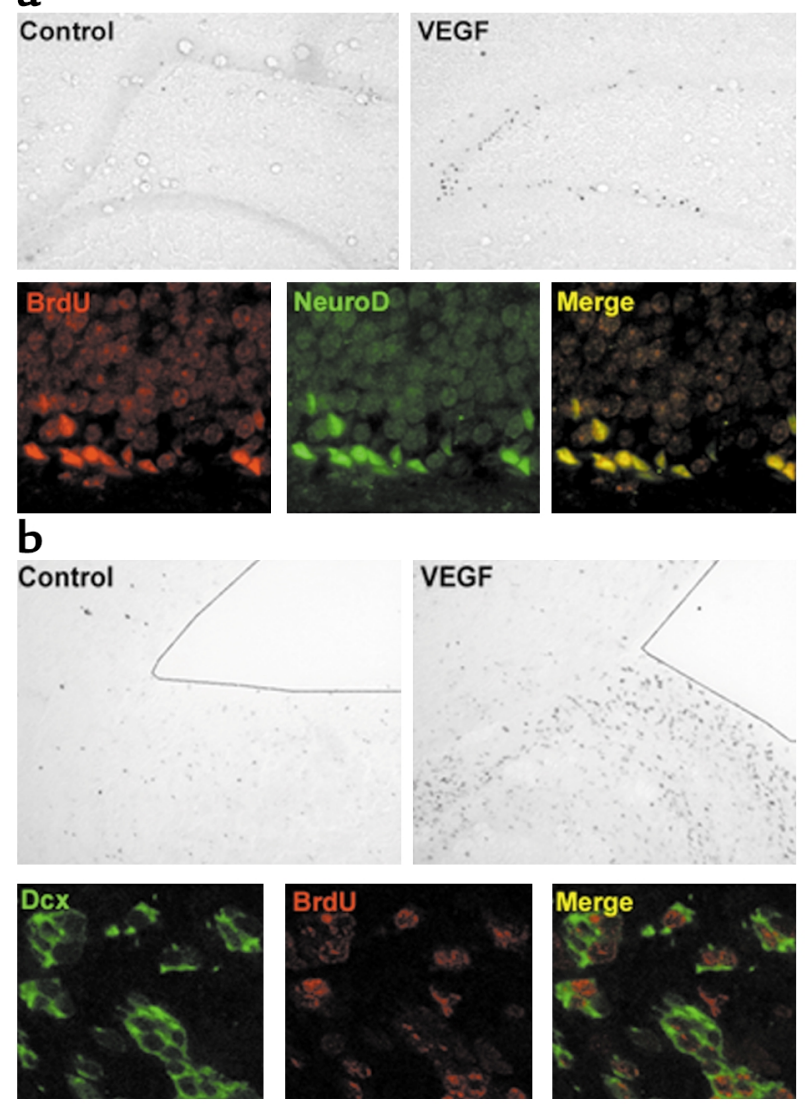

b

VEGF and neurogenesis in ischemic brain. To study VEGFinduced neurogenesis in relation to cerebral ischemia, rats were subjected to MCAO, given VEGF and the cellproliferation marker BrdU on days 1-3 after ischemia, and sacrificed at intervals thereafter (Figure 3). We showed previously that after VEGF infusion, BrdU incorporation in the two principal neuroproliferative zones of the mammalian brain - the subgranular zone (SGZ) of the hippocampal DG and the rostral subventricular zone (SVZ) - colocalizes with the neuronal lineage marker, Dcx (26). As found previously (27), MCAO alone increased BrdU labeling in both DG and SVZ and on

\section{Figure 5}

Effects of intraventricular VEGF on the number of BrdU-labeled neurons in DG and SVZ, ipsilateral and contralateral to MCAO, at 3 and 28 days after ischemia. For control, aCSF was administered instead of VEGF. Dotted line, basal level of BrdU labeling in normal, nonischemic rats. Two-way ANOVA showed significant effects of location $(\mathrm{F}=26.85, P<0.0001)$ but not treatment $(\mathrm{F}=2.48, P=0.120)$ on the number of BrdU-labeled neurons at 3 days after ischemia and of location $(\mathrm{F}=22.13, P<0.0001)$ and treatment $(\mathrm{F}=27.28, P<0.0001)$ on the number of BrdU-labeled neurons at 28 days after ischemia, but no interaction between location and treatment at 3 days $(\mathrm{F}=1.14, P=0.340)$ or 28 days $(\mathrm{F}=3.83, P=0.014) .{ }^{*} P<0.05$, ${ }^{*} P<0.01$ compared with control on same day at same location (Tukey test; $n=9$ ). DG-I, ipsilateral dentate gyrus; DG-C, contralateral dentate gyrus; SVZ-I, ipsilateral subventricular zone; SVZ-C, contralateral subventricular zone.

\section{Figure 4}

Colocalization of BrdU and markers of neuronal lineage in DG (a) and SVZ (b) of the VEGF-treated, postischemic rat brain. Rats underwent $\mathrm{MCAO}$ and were treated with intraventricular aCSF (Control) or VEGF and killed 28 days after MCAO. ( $\mathbf{a}$ and $\mathbf{b}$ ) Brain sections were immunostained for BrdU (top) or for both BrdU and the neuronal lineage marker NeuroD (a) (bottom) or Dcx (b) (bottom). VEGF treatment increased BrdU labeling in both DG and SVZ, and BrdU labeling colocalized with neuronal lineage markers in both regions. Lines in upper panels of $\mathbf{b}$ demarcate the lateral ventricle. Data shown are representative of findings from at least four animals per experiment.

both the ipsilateral (ischemic) and contralateral (control nonischemic) sides of the brain (Figures 4 and 5). In each case, the number of BrdU-labeled cells was 1.5- to 2-fold higher on the ischemic side. In both DG and SVZ, VEGF produced no further increase in BrdU labeling beyond that caused by ischemia alone at 3 days, but at 28 days the number of BrdU-labeled cells was higher on both the ischemic and nonischemic side in VEGF-treated animals. The magnitude of this difference ranged from 2.7fold in the SVZ on the ischemic side to 9.3-fold in the DG on the nonischemic side. These findings indicate that (a) the neuroproliferative effects of ischemia and VEGF are not additive, suggesting that the effect of ischemia may be mediated by VEGF or by another factor that activates the same signal transduction pathways activated by VEGF; and (b) in contrast to the increase in BrdU labeling in DG and SVZ induced by VEGF in
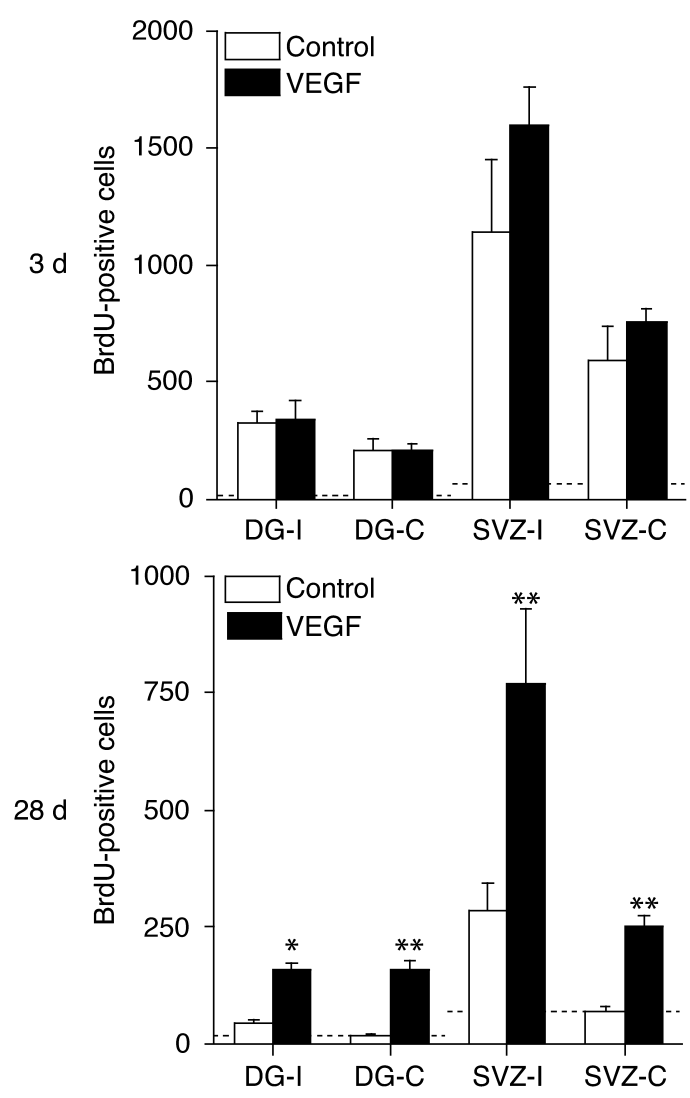


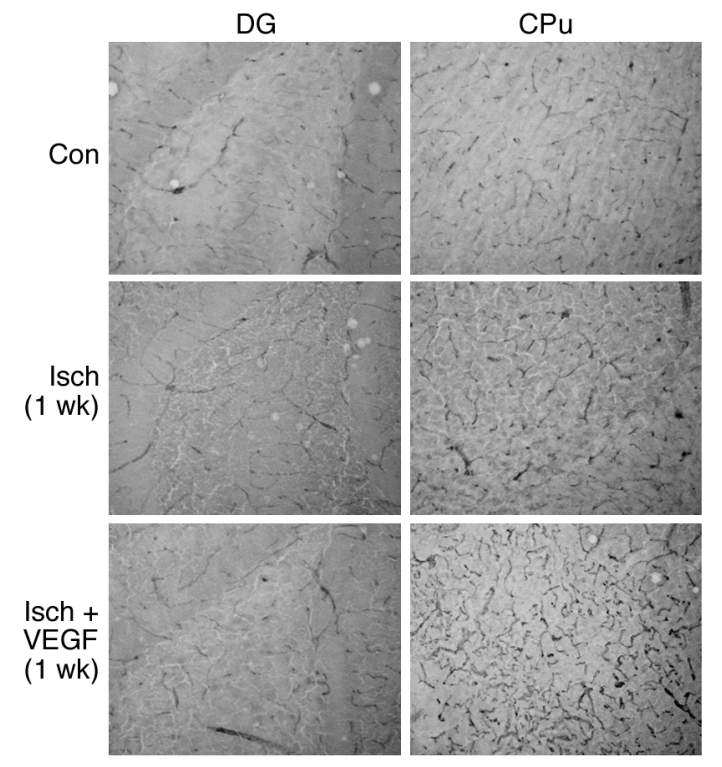

nonischemic brains at shorter intervals (26), the delayed emergence of the effect observed at 28 days suggests that it is likely to involve increased survival rather than increased proliferation of VEGF-stimulated precursors.

VEGF and angiogenesis in ischemic brain. VEGF stimulates cerebral angiogenesis in both the normal (10) and ischemic (13) brain, but this effect has not been examined in the brain's neuroproliferative zones. This is important because of the proposed relationship between angiogenesis and neurogenesis (24-26). To evaluate the effect of VEGF on angiogenesis in neuroproliferative zones of the ischemic rat brain, sections through DG (where a "vascular niche" for neurogenesis has been postulated; see ref. 24) were stained with an Ab against vWF, an endothelial cell marker expressed by VEGF-induced cerebral neovasculature (26). Compared with control brains, neither ischemia nor the combination of ischemia and VEGF treatment increased vWF staining in DG (Figures 6 and 7), despite the fact that both increased BrdU labeling in this region (Figure 5). In contrast, vWF staining was increased in the penumbral region of the ischemic striatum after ischemia plus VEGF treatment, consistent with prior evidence for VEGF-induced angiogenesis in ischemic brain areas (13).

\section{Discussion}

The major finding of this study is that the administration of VEGF to rats undergoing focal cerebral ischemia reduces infarct size and enhances neurogenesis and

\section{Figure 7}

Effects of intraventricular VEGF on the number of vWF-labeled cells in DG (top) and in the penumbral region of ischemic caudate-putamen (CPu) (bottom). For control, aCSF was administered instead of VEGF. Dotted line, basal level of BrdU labeling in normal, nonischemic rats. ${ }^{*} P<0.05$ compared with 0 days; ${ }^{\#} P<0.05$ compared with same-day control (ANOVA and post hoc Student-NewmanKeuls tests; $n=3-7$ ).

\section{Figure 6}

vWF immunohistochemistry in DG and caudate-putamen (CPu) from control rats (Con) and 1 week after MCAO (Isch) or MCAO plus VEGF treatment (Isch + VEGF). For the latter two groups, fields shown are from the ischemic hemisphere. Data shown are representative of findings from at least four animals per experiment.

cerebral angiogenesis. Of particular note, these effects were observed with delayed administration of VEGF, which was given beginning at day 1 and continued until day 3 after ischemia. This regimen was adopted because a previous study showed that delayed, but not early, postischemic administration of VEGF improved neurological recovery after stroke, apparently because early VEGF treatment promoted brain edema, hemorrhagic transformation of the infarct, and spread of the ischemic lesion (13). Neuroprotection following such delayed treatment is unusual, although it has been reported $(32,33)$. One explanation may be that most experimental neuroprotective agents have been directed against early events in ischemia, such as acute excitotoxicity, whereas VEGF may act on later events to prevent delayed cell death. In addition, in the model we employed, ischemia may evolve more slowly than it does after more prolonged $\mathrm{MCAO}$, thereby expanding the time window for cell rescue. VEGF also produced a strikingly delayed effect on neurogenesis, which was apparent only after 28 days. This suggests a mechanism of action that, while triggered within the first 3 days after ischemia, requires considerably longer to become manifest.

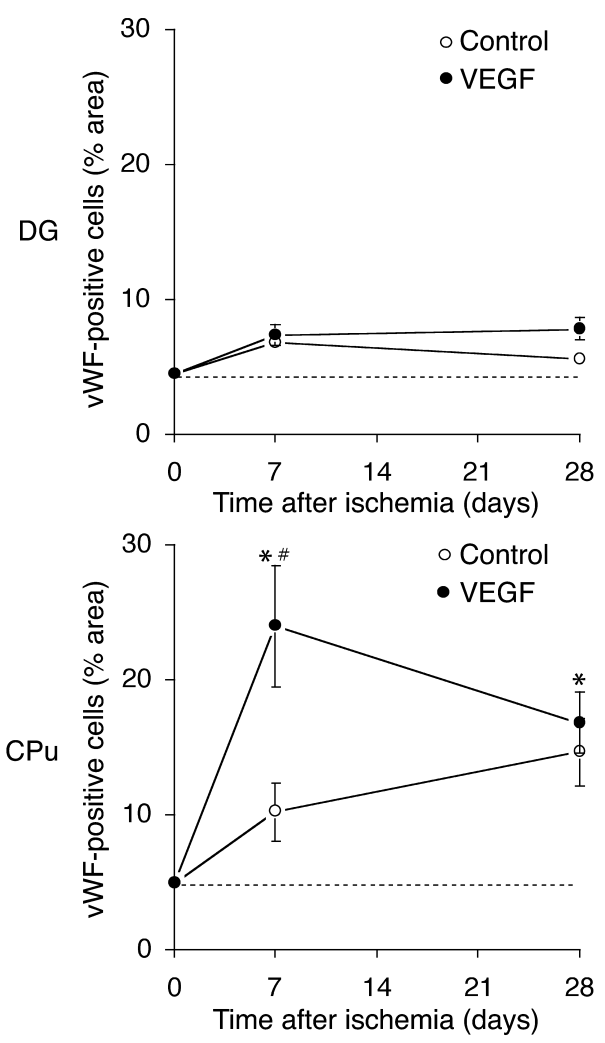


VEGF and neuroprotection in ischemic brain. At least four previous studies have examined the effect of VEGF on cerebral infarct size. Hayashi et al. (12) applied VEGF topically to the surface of the rat brain after 90 minutes of MCAO, followed by reperfusion, and found that infarct volume at 24 hours was reduced approximately $45 \%$. Bao et al. (14) used a different approach, in which transient MCAO in the rat was followed by intraventricular infusion of an antiVEGF Ab for 7 days; in this case, the Ab increased infarct volume approximately $35 \%$, providing indirect evidence for a role of VEGF in limiting infarct size. In contrast, van Bruggen et al. (34) reported that intraperitoneal administration of $\operatorname{mFLT}(1-3)$-IgG, a fusion protein that sequesters VEGF, led to a decrease in infarct size of approximately $30 \%$ at $8-12$ weeks after MCAO in mice. Finally, Zhang et al. (13) found a biphasic effect, wherein intravenous administration of VEGF to rats increased infarct size approximately $25 \%$ at 9 days when given 1 hour after MCAO, but improved neurological function at 7-28 days when given 48 hours after MCAO. These different results are not surprising considering that the studies cited differ with respect to species, whether MCAO was followed by reperfusion, whether VEGF or a VEGFbinding protein was given, the route and timing of administration, duration of follow up, and whether histological or functional criteria were used to evaluate outcome. One major determinant of the effect of VEGF on the ischemic brain (and a likely source of variability in the studies mentioned above) is the prominence of VEGF-induced edema, which is related to VEGF's ability to enhance vascular permeability $(13,34)$. Our finding that intraventricular VEGF reduces infarct volume and improves neurological outcome at 3-28 days after transient focal ischemia is most consistent with the studies by Hayashi et al. (12) and Bao et al. (14), and with the delayed administration protocol used by Zhang et al. (13), and suggests that under the proper conditions, VEGF can protect the brain against ischemia. Although the mechanisms underlying VEGF-induced reduction of infarct size are uncertain, several lines of evidence, cited above, point to a direct protective effect on neurons. In previous studies, we found that VEGF reduced neuronal death from hypoxia in vitro and that this involved activation of the VEGFR2/ Flk1 receptor and $\mathrm{PI} 3 \mathrm{~K}$ and decreased activation of caspase-3 $(19,20)$.

VEGF and neurogenesis in ischemic brain. Palmer et al. (24) first proposed a role for VEGF in neurogenesis, observing that in the DG new neurons were often observed in the vicinity of blood vessels. Louissaint et al. (25) went on to show that in the adult songbird brain, testosterone-induced angiogenesis was associated with increased production of brain-derived neurotrophic factor by endothelial cells and that an inhibitor of the VEGFR2/Flk1 tyrosine kinase blocked both the angiogenesis- and neurogenesis-promoting effects of testosterone in the higher vocal center of the canary brain. We reported that VEGF stimulated neurogenesis both in mouse brain cultures in vitro and in neuroproliferative regions (SVZ and SGZ) of the nonischemic mouse brain in vivo (26). In the latter case, VEGF was infused by the intracerebroventricular route for 3 days, BrdU was given by the intraperitoneal route over the same period, and neurogenesis was measured 1 week later. The observed increase in BrdU labeling of cells that coexpressed neuronal markers suggested that VEGF acted at an early (proliferative) phase of neurogenesis.

In the present study, we found that VEGF had a delayed effect on the number of BrdU-labeled cells of neuronal lineage in the ischemic brain, consistent with increased survival of newborn neurons rather than increased proliferation. This discrepancy can be reconciled by considering that focal cerebral ischemia itself induces neuroproliferation in SVZ and SGZ (27). Thus, the neuroproliferative effect of VEGF that we observed in the nonischemic brain (27) may be masked by the effect of ischemia itself in the ischemic brain. Comparison of control and VEGF-treated ischemic brain, however, reveals another effect of VEGF: the delayed appearance, between 3 and 28 days, of a difference between the number of BrdU-labeled cells of neuronal lineage in both SVZ and SGZ, and on both sides of the brain, with more such cells surviving in VEGF-treated mice in each case. This difference implies that VEGF not only stimulates the proliferation of neuronal precursors in the nonischemic brain, but also promotes the survival of proliferating cells of neuronal lineage in the ischemic brain, consistent with the neurotrophic effects of VEGF demonstrated previously in other systems (15-18). Because VEGF was administered for only 3 days following ischemia, even the delayed effects of VEGF must have been triggered during this period. Previous studies on the role of trophic factors in neurogenesis provide clues as to how this might occur $(35,36)$. Thus, the VEGFinduced increase in survival of neuronal precursor cells could be due to either long latency, downstream effects of VEGF signaling in the same cells that are stimulated to proliferate by ischemia (including priming these cells for the subsequent effects of other growth factors), or by enhanced proliferation of a separate, VEGF-responsive subpopulation characterized by more robust survival capacity.

$V E G F$ and angiogenesis in ischemic brain. The timing and distribution of VEGF expression correlate with angiogenesis in the normal and hypoxic developing adult rat brain (4) and the ischemic adult (9) rat brain. For example, VEGF protein expression in the ischemic penumbra increased progressively between 2 and 14 days after MCAO, and evidence of new vessel formation was present during days 7-28 (9). Intracerebral infusion of VEGF stimulates angiogenesis detectable by laminin immunostaining in rat cerebral cortex $3-7$ days later $(10,11)$. In the ischemic 
rat brain, intravenously administered VEGF also triggers angiogenesis, as manifested by an increase in the number and volume of FITC-dextran-perfused cerebral cortical microvessels 7 days later (13). We found an increase in the density of vWF-immunoreactive blood vessels 7-28 days after focal ischemia plus VEGF treatment in the striatal ischemic penumbra. The vWF immunostaining increased roughly fourfold over control levels and roughly twofold over levels in ischemic penumbra of rats not given VEGF. Neither ischemia nor ischemia plus VEGF significantly affected vessel density in the DG, however. This may be because (a) the DG does not undergo ischemic injury in this model, as shown by cresyl violet and Klenow staining (27), and (b) an ischemia-induced increase in the expression of VEGF receptors may be required to maximize the angiogenic effect of VEGF $(5,7,37)$. In the present context, the failure of ischemia or VEGF to stimulate angiogenesis in DG argues that angiogenesis is not required for the neuroproliferative response to ischemia or VEGF in this region, although angiogenesis might still promote neurogenesis from resident precursor cells in the ischemic penumbra.

Several studies have shown that cerebral ischemia increases VEGF expression $(5,6,38-40)$, so it may be puzzling that administration of exogenous VEGF should be able to modify infarct size, neurogenesis, or angiogenesis in the ischemic brain. This apparent paradox may be explained by differences in concentration, distribution, or timing of exposure to endogenous and exogenous VEGF. For example, intraventricular delivery of exogenous VEGF, as in this study, may result in higher levels of VEGF in the vicinity of the brain's neuroproliferative zones because of the proximity of both SVZ and DG to the lateral ventricles.

In conclusion, our findings suggest that in the ischemic rat brain, administration of exogenous VEGF (a) provides an early neuroprotective effect that reduces infarct size and improves neurological outcome, which is maximal by 3 days; (b) promotes the survival of nascent neurons arising in DG and SVZ, an effect that becomes evident between 3 and 28 days; and (c) stimulates angiogenesis in the ischemic penumbra, but not in neuroproliferative zones remote from the site of ischemia. Thus, direct neuroprotection may reduce ischemic injury in the acute phase, whereas neurogenesis, angiogenesis, or both may contribute to longerterm repair of the injured brain. For example, neurogenesis might have the capacity to enhance brain repair after ischemia by replacement of dead cells, and both neurogenesis and angiogenesis could lead to the release of growth factors that promote recovery indirectly. Moreover, the effects of VEGF on neurogenesis and angiogenesis could be interrelated: although we did not observe ischemia-induced angiogenesis in DG, its occurrence in the ischemic penumbra might promote neurogenesis from resident neuronal precursor cells. These findings may have implications for the possible role of VEGF as a therapeutic agent for stroke.

\section{Acknowledgments}

This work has been supported by a United States Public Health Service grant (NS44921).

1. Leung, D.W., Cachianes, G., Kuang, W.-J., Goeddel, D.V., and Ferrara, N. 1989. Vascular endothelial growth factor is a secreted angiogenic mitogen. Science. 246:1306-1309.

2. Keck, P.J., et al. 1989. Vascular permeability factor, an endothelial cell mitogen related to PDGF. Science. 246:1309-1312.

3. Shweiki, D., Itin, A., Soffer, D., and Keshet, E. 1992. Vascular endothelial growth factor induced by hypoxia may mediate hypoxia-initiated angiogenesis. Nature. 359:843-845.

4. Ogunshola, O.O., et al. 2000. Neuronal VEGF expression correlates with angiogenesis in postnatal developing rat brain. Brain Res. Dev. Brain Res. 119:139-153.

5. Kovàcs, Z., Ikezaki, K., Samoto, K., Inamura, T., and Fukui, M. 1996. VEGF and flt: expression time kinetics in rat brain infarct. Stroke. 27:1865-1873.

6. Hayashi, T., Abe, K., Suzuki, H., and Itomaya, Y. 1997. Rapid induction of vascular endothelial growth factor gene expression after transient middle cerebral artery occlusion in rats. Stroke. 28:2039-2044.

7. Lennmyr, F., Ata, K.A., Funa, K., Olsson, Y., and Terent, A. 1998. Expression of vascular endothelial growth factor (VEGF) and its receptors (Flt-1 and Flk-1) following permanent and transient occlusion of the middle cerebral artery in the rat. J. Neuropathol. Exp. Neurol. 57:874-882.

8. Plate, K.H., Beck, H., Danner, S., Allegrini, P.R., and Wiessner, C. 1999. Cell type specific upregulation of vascular endothelial growth factor in an MCA-occlusion model of cerebral infarct. J. Neuropathol. Exp. Neurol. 58:654-666.

9. Zhang, Z.G., et al. 2002. Correlation of VEGF and angiopoietin expression with disruption of blood-brain barrier and angiogenesis after focal cerebral ischemia. J. Cereb. Blood Flow Metab. 22:379-392.

10. Rosenstein, J.M., Mani, N., Silverman, W.F., and Krum, J.M. 1998. Patterns of brain angiogenesis after vascular endothelial growth factor administration in vitro and in vivo. Proc. Natl. Acad. Sci. U. S. A. 95:7086-7091.

11. Krum, J.M., Mani, N., and Rosenstein, J.M. 2002. Angiogenic and astroglial responses to vascular endothelial growth factor administration in adult rat brain. Neuroscience. 110:589-604.

12. Hayashi, T., Abe, K., and Itoyama, Y. 1998. Reduction of ischemic damage by application of vascular endothelial growth factor in rat brain after transient ischemia. J. Cereb. Blood Flow Metab. 18:887-895.

13. Zhang, Z.G., et al. 2000. VEGF enhances angiogenesis and promotes blood-brain barrier leakage in the ischemic brain. J. Clin. Invest. 106:829-838.

14. Bao, W.L., Lu, S.D., Wang, H., and Sun, F.Y. 1999. Intraventricular vascular endothelial growth factor antibody increases infarct volume following transient cerebral ischemia. Chung Kuo Yao Li Hsueb Pao. 20:313-318.

15. Sondell, M., Lundborg, G., and Kanje, M. 1999. Vascular endothelial growth factor has neurotrophic activity and stimulates axonal outgrowth, enhancing cell survival and Schwann cell proliferation in the peripheral nervous system. J. Neurosci. 19:5731-5740.

16. Sondell, M., Sundler, F., and Kanje, M. 2000. Vascular endothelial growth factor is a neurotrophic factor which stimulates axonal outgrowth through the flk-1 receptor. Eur. J. Neurosci. 12:4243-4254.

17. Silverman, W.F., Krum, J.M., Mani, N., and Rosenstein, J.M. 1999. Vascular, glial and neuronal effects of vascular endothelial growth factor in mesencephalic explant cultures. Neuroscience. 90:1529-1541.

18. Jin, K.L., Mao, X.O., and Greenberg, D.A. 2000. Vascular endothelial growth factor rescues HN33 neural cells from death induced by serum withdrawal. J. Mol. Neurosci. 14:197-203.

19. Jin, K.L., Mao, X.O., and Greenberg, D.A. 2000. Vascular endothelial growth factor: direct neuroprotective effect in in vitro ischemia. Proc. Natl. Acad. Sci. U. S. A. 97:10242-10247.

20. Jin, K., et al. 2001. Caspase-3 and the regulation of hypoxic neuronal death by vascular endothelial growth factor. Neuroscience. 108:351-358.

21. Matsuzaki, H., et al. 2001. Vascular endothelial growth factor rescues hippocampal neurons from glutamate-induced toxicity: signal transduction cascades. FASEB J. 15:1218-1220.

22. Svensson, B., et al. 2002. Vascular endothelial growth factor protects cultured rat hippocampal neurons against hypoxic injury via an antiexcitotoxic, caspase-independent mechanism. J. Cereb. Blood Flow Metab. 22:1170-1175.

23. Oosthuyse, B., et al. 2001. Deletion of the hypoxia-response element in the vascular endothelial growth factor promoter causes motor neuron degeneration. Nat. Genetics. 28:131-138.

24. Palmer, T.D., Willhoite, A.R., and Gage, F.H. 2000. Vascular niche for adult hippocampal neurogenesis. J. Comp. Neurol. 425:479-494.

25. Louissaint, A., Rao, S., Leventhal, C., and Goldman, S.A. 2002. Coordinated 
interaction of neurogenesis and angiogenesis in the adult songbird brain. Neuron. 34:945-960.

26. Jin, K., et al. 2002. Vascular endothelial growth factor (VEGF) stimulates neurogenesis in vitro and in vivo. Proc. Natl. Acad. Sci. U. S. A. 99:11946-11950.

27. Jin, K., et al. 2001. Neurogenesis in dentate subgranular zone and rostra subventricular zone after focal cerebral ischemia in the rat. Proc. Natl. Acad. Sci. U. S. A. 98:4710-4715.

28. Longa, E.Z., Weinstein, P.R., Carlson, S., and Cummins, R. 1989. Reversible middle cerebral artery occlusion without craniectomy in rats. Stroke. 20:84-91.

29. Li, Y., et al. 2001. Treatment of stroke in rat with intracarotid administration of marrow stromal cells. Neurology. 56:1666-1672.

30. Swanson, R.A., et al. 1990. A semiautomated method for measuring brain infarct volume. J. Cereb. Blood Flow Metab. 10:290-293.

31. Jin, K., et al. 1999. In situ detection of neuronal DNA strand breaks using the Klenow fragment of DNA polymerase I reveals different mechanisms of neuron death after global cerebral ischemia. J. Neurochem. 72:1204-1214.

32. Heath, D.L., and Vink, R. 1999. Improved motor outcome in response to magnesium therapy received up to 24 hours after traumatic diffuse axonal brain injury in rats. J. Neurosurg. 90:504-509.

33. Candelario-Jalil, E., et al. 2002. Neuroprotective efficacy of nimesulide against hippocampal neuronal damage following transient forebrain ischemia. Eur. J. Pharmacol. 453:189-195.
34. van Bruggen, N., et al. 1999. VEGF antagonism reduces edema formation and tissue damage after ischemia/reperfusion injury in the mouse brain. J. Clin. Invest. 104:1613-1620.

35. Morrison, S.J., et al. 2000. Transient Notch activation initiates an irreversible switch from neurogenesis to gliogenesis by neural crest stem cells. Cell. 101:499-510.

36. Simpson, P.J., et al. 2002. Atrial natriuretic peptide type C induces a cellcycle switch from proliferation to differentiation in brain-derived neurotrophic factor- or nerve growth factor-primed olfactory receptor neurons. J. Neurosci. 22:5536-5551.

37. Jin, K.L., Mao, X.O., Nagayama, T., Goldsmith, P.C., and Greenberg, D.A. 2000. Induction of vascular endothelial growth factor receptors and phosphatidylinositol 3'-kinase/Akt signaling by global cerebral ischemia in the rat. Neuroscience. 100:713-717.

38. Cobbs, C.S., Chen, J., Greenberg, D.A., and Graham, S.H. 1998. Vascular endothelial growth factor expression in rat focal cerebral ischemia. Neurosci. Lett. 249:79-82.

39. Pichiule, P., Chavez, J.C., Xu, K., and LaManna, J.C. 1999. Vascular endothelial growth factor upregulation in transient global ischemia induced by cardiac arrest and resuscitation in rat brain. Brain Res. Mol. Brain Res. 74:83-90.

40. Jin, K.L., Mao, X.O., Goldsmith, P.C., Nagayama, T., and Greenberg, D.A. 2000. Induction of vascular endothelial growth factor and hypoxiainducible factor- $1 \alpha$ by global ischemia in rat brain. Neuroscience. 99:577-585. 